\title{
Electrophysiological Mapping of the Temporal Branch of the Facial Nerve
}

\author{
Hiroshi Tokimura ${ }^{1}$ Masashi Hirabaru${ }^{1}$ Seiji Miyajima ${ }^{2}$ Kenichiro Tajitsu ${ }^{2}$ Hitoshi Yamahata ${ }^{1}$ \\ Sei Sugata ${ }^{1}$ Kazunori Arita ${ }^{1}$
}

${ }^{1}$ Division of Neurosurgery, Department of Neurosurgery, Graduate School of Medical and Dental Sciences, Kagoshima University, Sakuragaoka, Kagoshima City, Japan

2 Division of Neurosurgery, Department of Neurosurgery, Sendai Shimin Hospital, Satsumasendai, Japan

J Neurol Surg A 2014;75:116-119.
Address for correspondence Dr. Hiroshi Tokimura, MD, Department of Neurosurgery, Graduate School of Medical and Dental Sciences, Kagoshima University, 8-35-1 Sakuragaoka, Kagoshima City, 890-8544, Japan (e-mail: hiroshitok@nag.bbiq.jp).

\begin{abstract}
Background Minimized frontal and frontolateral approaches have been proposed for the clipping of aneurysms and for removal of deep-seated supra- and parasellar lesions. To avoid postoperative facial nerve palsy, anatomical studies have been conducted; however, only one electrophysiological investigation has been reported. We studied the course of the facial nerve in the temporal region and identify the optimal pterional keyhole craniotomy from the perspective of facial nerve protection.

Materials and Methods We stimulated the temporal branch of the facial nerve in nine healthy volunteers and identified its course electrophysiologically. Electromyographic (EMG) responses were recorded from the superior orbicularis oculi, corrugator, and frontal muscles. The course of the temporal branch of the facial nerve was identified by tracking the stimulation points that elicited the best EMG responses.

Results and Conclusion The distances from the tragus to the canthus, the tragus to the

Keywords

- facial nerve

- neurophysiology

- pterional approach

- keyhole

- aneurysm

- clipping point where the facial nerve crosses over the zygomatic arch, the tragus to the first nerve bifurcation, the tragus to the second nerve bifurcation, and from the orbit to the middle rami were $80.8 \pm 1.8 \mathrm{~mm}, 40.2 \pm 1.6 \mathrm{~mm}, 58.1 \pm 2.8 \mathrm{~mm}, 79.1 \pm 3.4 \mathrm{~mm}$, and $16.4 \pm 0.9 \mathrm{~mm}$, respectively. We found that the temporal branch of the facial nerve innervates each muscle in posterior-anterior direction. Based on these results, we prefer the pterional keyhole approach and incise the skin at a site posterior to the temporal branch of the facial nerve.
\end{abstract}

\section{Introduction}

The pterional approach established by Yasargil et al ${ }^{1}$ to clip anterior circulation aneurysms has been the gold standard for more than 30 years. However, the supraorbital keyhole approach, first reported by Perneczkey in 1998, is less invasive and yields good surgical results. ${ }^{2}$ It provides for wide intracranial exposure in patients with deep-seated supra- and parasellar lesions ${ }^{3}$ and for sufficient space for microscopic manipulation. ${ }^{2,3}$ Subsequently, Hernesniemi et al ${ }^{4}$ and Cheng et $\mathrm{al}^{5}$ proposed the lateral supraorbital and pterional keyhole approach, respectively. Since 2001, we have used the pterional keyhole approach to clip anterior circulation aneurysms in more than 300 patients and obtained favorable outcomes.

Although frontal and frontolateral keyhole craniotomies appear to be less invasive, more efficient, and safe, they raise the possibility of iatrogenic injury to the temporal branch of the facial nerve because of its anatomical location. The received

June 6, 2011

accepted after revision

February 4, 2013

published online

August 12, 2013 (c) 2014 Georg Thieme Verlag KG Stuttgart · New York
DOI http://dx.doi.org/ 10.1055/s-0033-1343981. ISSN 2193-6315. 
occurrence of facial nerve palsy in patients undergoing supraorbital keyhole craniotomy has been reported. ${ }^{6}$

To avoid facial nerve palsy in patients treated by keyhole craniotomies, the surgical anatomy of the temporal branch of the facial nerve has been studied, ${ }^{7-11}$ and one electrophysiological investigation has been reported. ${ }^{12}$

\section{Materials and Methods}

Our study population consisted of nine healthy volunteers (four women, five men) ranging in age from 23 to 62 years $(39.1 \pm 4.2$ [mean \pm standard error of the mean]). All gave informed consent before entry into the study; our institutional ethics committee approved the procedures.

\section{Electromygraphic Recording}

With the volunteers in the supine position, surface electromyographies (EMGs) were recorded unilaterally from the superior orbicularis oculi, corrugator, and frontal muscles using 0.9-cm diameter silver/silver chloride surface electrodes. The active electrode was placed over each motor point, with the reference electrode over the nasion. Responses were recorded with an MEB4204 instrument (Nihon Kohden Corp., Tokyo) using three channels with high and low gains; they were filtered with a time constant of $3 \mathrm{msec}$ through a highpass filter set at $3000 \mathrm{~Hz}$.

\section{Electric Stimulation}

The facial nerves were stimulated with monophasic square pulses of $200 \mu$ sec duration. Stimuli were delivered with a constant current stimulator attached over the skin with the cathode placed on the optimal point for eliciting responses from each muscle; the anode was placed distal to the cathode so that the current flowed along the course of the facial nerve. The stimulus intensity was gradually increased to a supramaximal level to recruit all nerve fibers.

\section{Landmarks and Distances}

To study the entire course of the facial nerve in the temporal region, we used four landmarks (-Fig. 1). They were the zygomatic arch, orbit, tragus, and canthus. Using these landmarks, we measured the distance from the tragus to the canthus, from the tragus to the point where the facial nerve crosses the zygomatic arch, from the tragus to the first and second bifurcation of the temporal branch of the facial nerve, and from the orbit to the middle rami of the facial nerve. These five distances were recorded for all volunteers and averaged.

\section{Results}

EMGs were successfully recorded from all subjects. As shown in - Fig. 2, triphasic waveforms and compound muscle action potentials were recorded from each muscle. The averaged distance from the tragus to the canthus was $80.8 \pm 1.8 \mathrm{~mm}$; it was $40.2 \pm 1.6 \mathrm{~mm}$ from the tragus to the crossing over the zygomatic arch, $58.1 \pm 2.8 \mathrm{~mm}$ from the tragus to the first and $79.1 \pm 3.4 \mathrm{~mm}$ to the second bifurcation, and

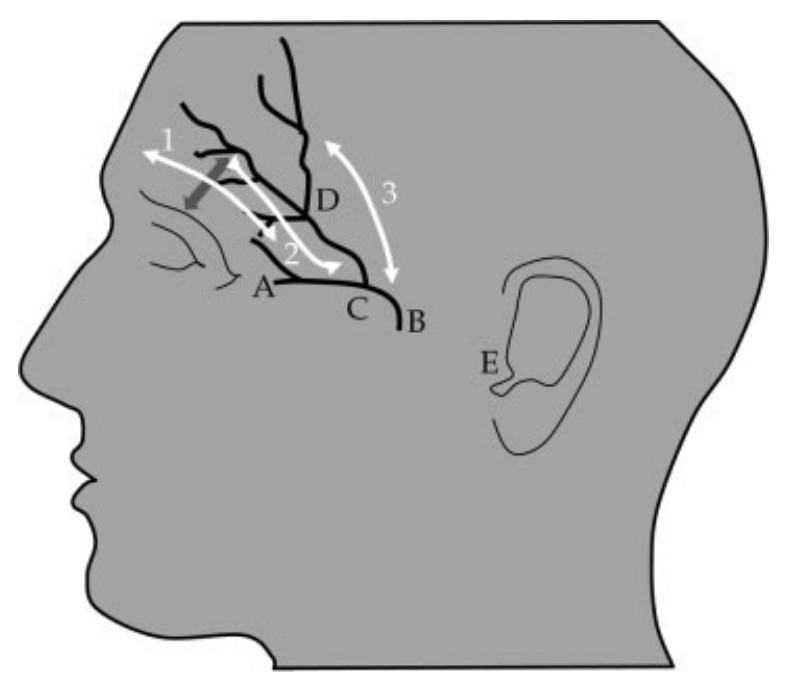

Fig. 1 Landmarks of the temporal branch of the facial nerve and relationship between the facial nerve and skin incisions of keyhole craniotomies, including the cathus $(A)$ crossing point over the zygomatic arch (B), first bifurcation (C), second bifurcation (D), and tragus (E). 1 = supraorbital; 2 = outer canthal; 3 = pterional keyhole craniotomy. We measured the distance between the orbit and the middle rami of the facial nerve.

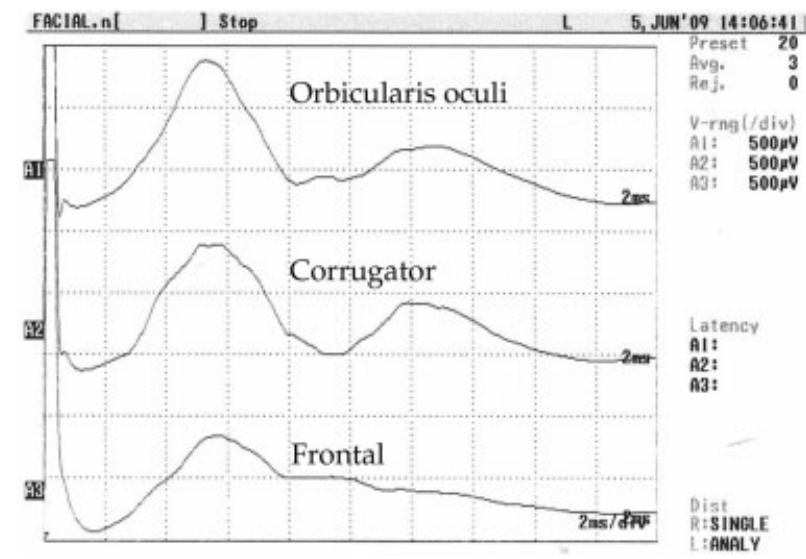

Fig. 2 Raw wave of compound muscle action potentials evoked by electrical stimulation of the facial nerve at each point from the orbicularis oculi (upper trace), corrugator (middle trace), and frontal muscle (lower trace).

$16.4 \pm 0.9 \mathrm{~mm}$ from the orbit to the middle rami. Stimulation in the area anterior to the middle rami evoked muscle responses from corrugator and superior orbicularis oculi muscles, indicating that the temporal branch of the facial nerve innervates each muscle in a posterior-anterior direction.

To avoid bias from far field activity of adjacent muscles, needle electrodes should be used for muscle recordings. However, because our study subjects were awake healthy volunteers, we used surface electrodes. Although there were some small effects from adjacent muscles, upon stimulation of the middle rami of the temporal branch of the facial nerve, 
we were able to recognize all responses from the orbicularis oculi muscle.

\section{Discussion}

Although the pterional approach ${ }^{1}$ has been the gold standard to clip anterior circulation aneurysms, it necessitates a semicoronal long skin incision from a point near the tragus to the midline in the hairline. However, the clipping of common anterior circulation aneurysms does not require such a large craniotomy; a small bone window appropriately placed on the Sylvian fissure usually suffices. A cosmetic reason for using a large craniotomy is to hide the incision behind the hairline; another reason is to avoid facial nerve injury. However, this technique may result in postoperative numbness of the skin in the parietal region and may result in a long alopecia zone along the skin incision, atrophy of the temporal muscle, limited mouth opening ability because of cutting of the temporal muscle, and skull deformity.

Three less invasive surgical approaches-the supraorbit$\mathrm{al}^{2,13}$ lateral supraorbital, ${ }^{4,14,15}$ and pterional keyhole approach $^{5}$-have been proposed to overcome the disadvantages of the pterional approach. They are characterized by a small craniotomy in the frontotemporal area, the site of the temporal branch of the facial nerve. To avoid facial nerve palsy after frontotemporal craniotomy, the surgical anatomy of the temporal branch of the facial nerve has been studied. ${ }^{7-11,15}$ These studies showed that the temporal branch of the facial nerve resides in a 15 - to $44-\mathrm{mm}$ region measuring from the orbit, the zygomatic suture, and the anterior margin of the zygomatic arch and that a region within $15 \mathrm{~mm}$ of the supralateral to the orbit represents a safe zone. Furnas ${ }^{11}$ recommends staying above the temporal root of the zygoma, $1 \mathrm{~cm}$ posterosuperior to the anterior hairline at the zygomatic arch, and $2 \mathrm{~cm}$ posterosuperior to the lateral edge of the eyebrow.

As shown in - Fig. 2, with respect to positional relationships, the supraorbital keyhole craniotomy ${ }^{2}$ is located anterior to the middle rami, the lateral supraorbital keyhole craniotomy $^{4,15}$ is placed between the anterior and middle rami, and the pterional keyhole craniotomy ${ }^{5}$ is situated posterior to the whole temporal branch of the facial nerve. In our experience, supraorbital keyhole craniotomies sometimes result in postoperative facial nerve palsy, which resolves in about 6 months. Lan et $\mathrm{al}^{6}$ reported a patient who presented with descensus of the eyebrow 3 months after undergoing a supraorbital keyhole craniotomy.

Park $^{12}$ reported that preoperative facial nerve mapping facilitates visualization of the entire nerve course on the skin and permits a more aggressive approach in dealing with facial skin and muscle flaps while reducing the risk of nerve injury. Our electrophysiological study of the temporal branch of the facial nerve showed that the distance from the orbit to the middle rami was $16.4 \mathrm{~mm}$, a finding consistent with anatomical studies. ${ }^{7-11,15}$ We also document that muscle responses from corrugator- and superior orbicularis oculi muscles were evoked by stimulation in the area anterior to the middle rami. This indicates that the temporal branch of the facial nerve innervates each muscle in posterior-anterior direction and that the supra- to lateral orbital area may not constitute a safe zone. We found that the zygomatic arch at around $40 \mathrm{~mm}$ from the tragus runs in the superoanterior direction and innervates muscles in a posterior-anterior direction. Although this distance is longer than reported by Miloro et $\mathrm{al}^{16}{ }^{16}$ the difference is attributable to the use of different reference points. Whereat they measured from the most anterior aspect of the bony external auditory canal, our measurements were from the tragus. The site of the skin incision for pterional keyhole craniotomy coincides with the anterior margin of the temporal hairline; it is always behind the temporal branch of the facial nerve. Therefore, in our opinion, pterional keyhole craniotomy can be expected to yield excellent cosmetic results, and it avoids iatrogenic injury of the temporal branch of the facial nerve. However, because there may be variations in the course of the facial nerve among patients, we strongly recommend electrophysiological mapping, possibly with polygraph instruments, to record motor evoked potentials before skin incision for keyhole pterional craniotomies.

\section{Conflict of Interest None}

\section{References}

1 Yasargil MG, Fox JL, Ray MW. Advances and Technical Standards in Neurosurgery: The Operative Approach to Aneurysms of the Anterior Communicating Artery. Vienna: Springer Verlag; 1975: $113-170$

2 van Lindert E, Perneczky A, Fries G, Pierangeli E. The supraorbital keyhole approach to supratentorial aneurysms: concept and technique. Surg Neurol 1998;49(5):481-489, discussion 489-490

3 Reisch R, Perneczky A. Ten-year experience with the supraorbital subfrontal approach through an eyebrow skin incision. Neurosurgery 2005;57(4, Suppl):242-255, discussion 242-255

4 Hernesniemi J, Ishii K, Niemela $M$, et al. Lateral supraorbital approach as an alternative to the classical pterional approach. Acta Neurochir (Wien) 2005;(Suppl 94):17-21

5 Cheng WY, Lee HT, Sun MH, Shen CC. A pterion keyhole approach for the treatment of anterior circulation aneurysms. Minim Invasive Neurosurg 2006;49(5):257-262

6 Lan Q Gong Z, Kang D, et al. Microsurgical experience with keyhole operations on intracranial aneurysms. Surg Neurol 2006; 66(Suppl 1):S2-S9

7 Gosain AK. Surgical anatomy of the facial nerve. Clin Plast Surg 1995;22(2):241-251

8 Salas E, Ziyal IM, Bejjani GK, Sekhar LN. Anatomy of the frontotemporal branch of the facial nerve and indications for interfascial dissection. Neurosurgery 1998;43(3):563-568, discussion 568-569

9 Bernstein L, Nelson RH. Surgical anatomy of the extraparotid distribution of the facial nerve. Arch Otolaryngol 1984;110 (3):177-183

10 Ammirati M, Spallone A, Ma J, Cheatham M, Becker D. An anatomicosurgical study of the temporal branch of the facial nerve. Neurosurgery 1993;33(6):1038-1043, discussion 1044

11 Furnas DW. Landmark for the trunk and the temporo-facial division of the facial nerve. Br J Surg 1965;52:694-696 
12 Park JI. Preoperative percutaneous facial nerve mapping. Plast Reconstr Surg 1998;101(2):269-277

13 Mitchell P, Vindlacheruvu RR, Mahmood K, Ashpole RD, Grivas A, Mendelow AD. Supraorbital eyebrow minicraniotomy for anterior circulation aneurysms. Surg Neurol 2005;63(1):4751, discussion 51

14 Mori K, Osada H, Yamamoto T, Nakao Y, Maeda M. Pterional keyhole approach to middle cerebral artery aneurysms through an outer canthal skin incision. Minim Invasive Neurosurg 2007;50 (4):195-201

15 Mori K, Yamamoto T, Nakao Y, et al. Lateral supraorbital keyhole approach to clip unruptured anterior communicating artery aneurysms. Minim Invasive Neurosurg 2008;51(5):292-297

16 Miloro M, Redlinger S, Pennington DM, Kolodge T. In situ location of the temporal branch of the facial nerve. J Oral Maxillofac Surg 2007;65(12):2466-2469 